\title{
Temperature and Volume Effects on Local Segmental Relaxation in Poly(vinyl acetate)
}

\section{M. Roland* and R. Casalini}

Naval Research Laboratory, Chemistry Division, Code 6120, Washington, D.C. 20375-5342

Received October 31, 2002; Revised Manuscript Received J anuary 6, 2003

\begin{abstract}
Dielectric data on the local segmental relaxation of amorphous poly(vinyl acetate) (PVAc) are analyzed. At atmospheric pressure, the fragility ( $T_{\mathrm{g}}$-normalized temperature dependence) is invariant to molecular weight. Since the fragility is also independent of pressure, the combined temperature and pressure dependences of the relaxation times can be analyzed using the Avramov model of structural relaxation. This entropy model gives a satisfactory description of the data and yields an expression for the pressure dependence of the dynamic glass transition identical to the empirical Andersson equation. Although in principle the Avramov model parameters can be deduced from measurable thermodynamic properties of the glass-former, the results are at odds with the values obtained from fitting the relaxation times for PVAc. Using pressure-vol ume-temperature data, the respective contributions of thermal energy and volume to the local dynamics in PVAc were assessed. Specifically, we calculate the ratio of the isochoric to isobaric apparent activation energies. The obtained value, $\approx 0.6$ at atmospheric pressure just above $\mathrm{T}_{\mathrm{g}}$, indicates that temperature and volume both govern to a significant degree the relaxation times. This result is supported by a comparison of the relative magnitude of the isobaric and isochronal thermal expansion coefficients. These properties of PVAc are similar to those of other nonassociated polymers and glass-forming liquids and can be shown to be weakly correlated with the degree of super-Arrhenius behavior.
\end{abstract}

\section{Introduction}

The transition of a liquid or polymeric melt into the glassy state can be effected by cooling as well as by the application of hydrostatic pressure. A striking feature of liquids approaching their glass temperature is the devel opment of a strongly non-Arrhenius temperature dependence of the relaxation times, $\tau$. Whereas at high temperature, local relaxation is a thermally activated process, near $T_{g}$, densification and the consequent molecular crowding effect strong intermolecular cooperativity (many-body interactions) and heterogeneous dynamics. Thefundamental mechanisms underlying the glass transition continue to be studied and vigorously debated. The topic is of obvious importance, including the fact that understanding the glass transition can provide better understanding of the glassy state itself.

Because glass-forming liquids and polymers display complex properties, a variety of methods have been brought to bear in investigations of the glass transition. Diel ectric spectroscopy is particularly applicable because the supercooled dynamics encompass such a broad frequency range. Data on small-molecule glasses and polymers differing in $\mathrm{T}_{\mathrm{g}}$ are commonly correlated using the $T_{g}$-normalized temperature dependence of the relaxation times (fragility, or steepness index, defined as $\left.\mathrm{m}=\mathrm{d} \log (\tau) /\left.\mathrm{d}\left(\mathrm{T}_{\mathrm{g}} / \mathrm{T}\right)\right|_{\left.\mathrm{T}=\mathrm{T}_{\mathrm{g}}\right) .}\right)^{1,2}$ In place of the glass temperature, it is more appropriate to use the temperature, $\mathrm{T}_{\alpha}$, at which the dielectric relaxation time assumes an arbitrary, long value, ca. $100 \mathrm{~s}$. This $\mathrm{T}_{\alpha}$ is expected to be close to the calorimetric or dilatometric $\mathrm{T}_{\mathrm{g}}$. The two characteristic temperatures have also been shown to have the same pressure dependence. ${ }^{3-6}$

The fragility method of classifying glass-formers derives from a configurational entropy approach to supercooled dynamics. ${ }^{7}$ Other schemes have been utilized, in particular plotting the relaxation times as a function of $T-T_{g}$, as suggested by free volume interpretations of the glass transition. 1,8,9 N ormalizing temperature by $\mathrm{T}_{\mathrm{g}}$ appears to be the correct method, if the sensitivity of $\tau$ to temperature is expected to reflect the strength of the intermol ecular cooperativity. ${ }^{10-12}$

The combination of traditional temperature experiments with measurements at el evated hydrostatic pressure is necessary to completely characterize the dynamic state. Such experiments, which yield an order of magnitude increase in the obtained information, rely for their interpretation on models of the combined pressure and temperature dependences of dielectric relaxation times. Fytas and co-workers generalized the VogelFulcher equation to include the effect of pressure..$^{13}$ However, the predicted proportional ity between $\mathrm{T}_{\mathrm{g}}$ and pressure is not generally found. ${ }^{14-17}$ The free volume model of Cohen and Grest ${ }^{18}$ provides an expression for $\tau(T, P)$, but experimental results deviate from the predicted form. ${ }^{19,20}$ Bendler and co-workers ${ }^{21}$ recently extended their defect diffusion model to consider the effect of pressure on $\tau$. Casalini et al. ${ }^{22}$ addressed pressure effects through an extension of the AdamGibbs entropy model. This leads to the prediction that the temperature dependence of $\tau$ (i.e., fragility) will vary with pressure, which is valid only for some materials. ${ }^{17,23}$ In contrast, the entropy model of Avramov ${ }^{24}$ explicitly treats temperature and pressure dependences as separable, whereby $\mathrm{m}$ is not a function of pressure.

An important aspect of theoretical investigations of the glass transition is the relative emphasis given to temperature and volume as control variables. Early literature focused on the role of free volume. $8,18,25,26 \mathrm{M}$ ore recently, the idea has been espoused that the supercool ed dynamics are governed by thermal energy, with any direct effects of volume discounted. ${ }^{27-29}$ Of course, potential energy barriers reflect local steric constraints, and likewise temperature-dependent parameters can be incorporated into a free volume model; 30,31 thus, strict 
distinctions between temperature and volume are to some extent pedantic. Nevertheless, it is of interest to quantify their relative contribution to the dynamics near $\mathrm{T}_{\mathrm{g}}$. Recent studies ${ }^{28,32,33}$ seem to indicate that, at least for hydrogen-bonded glass-formers, temperature primarily governs structural relaxation. However, this is not the case for nonassociated liquids and polymers, in which density exerts a very substantial role. ${ }^{34,35}$

In this paper we analyze literature data on the dielectric relaxation of poly(vinyl acetate) (PVAc), a chemically stable, commercially important material, commonly utilized in adhesives, coatings, and paints. PVAc has interesting structural variations and can be obtained in the atactic, and thus amorphous form, which is crucial for investigations of the glass transition. An ample dipole moment facilitates the use of dielectric spectroscopy. Early dielectric studies of PVAc were limited to relatively narrow frequency ranges. ${ }^{36} \mathrm{How}$ ever, the more recent measurements of Stickel ${ }^{37}$ and Richert ${ }^{38}$ encompass 16 decades of frequency, providing the most complete set of relaxation time data. Several investigators have al so studied the effect of pressure on the dielectric relaxation of PVAc, with the most extensive results due to Heinrich and Stoll. ${ }^{39}$

We analyze herein both the temperature and pressure dependence of the dielectric relaxation times of PVAC. Making use of published PVT data, we also calculate the volume dependence of the relaxation times. This enables apparent activation energies to be determined for both isochoric (constant volume) and isobaric conditions. These yield a direct measure of the degree to which volume and thermal energy govern the dynamics of PVAc at temperatures just above $T_{g}$.

\section{Results and Discussion}

Atmospheric Pressure Dielectric Relaxation Times. Two dielectric investigations of unusually broad range have been carried out on the same sample of amorphous PVAc. ${ }^{37,38}$ The results for the relaxation time, defined from the maximum in the dielectric loss (which is approximately the most probable relaxation time), are displayed in Figure 1 . There is a subtle change in the dynamics of PVAc at a temperature of $383 \mathrm{~K}$, associated with myriad property changes. ${ }^{37,40}$ However, the consequent deviation in $\tau(T)$ is weak and not of interest herein. The material used for these measurements had a molecular weight of $15000 \mathrm{~g} / \mathrm{mol}, 37$ which is bel ow the limiting values, at which the properties of PVAc become independent of molecular weight. ${ }^{36}$

Heinrich and Stoll ${ }^{39}$ used dielectric spectroscopy to study a higher molecular weight amorphous PVAC $\left(\mathrm{M}_{\mathrm{w}}=170000 \mathrm{~g} / \mathrm{mol}\right)$, with pressure included as a variable in their experiments. The results for $\mathrm{P}=0.1$ $\mathrm{MPa}$ are included in Figure 1, where it can be seen that both the magnitude of the relaxation times and their apparent temperature dependence are different for the two samples. From Figure 1, we obtain $\mathrm{T}_{\alpha}(\tau=100 \mathrm{~s})=$ 300.1 and $310.7 \mathrm{~K}$ respectively for the low and high molecular weight PVAc. These bracket the calorimetric $\mathrm{T}_{\mathrm{g}}$ of high molecular weight PVAc.4 ${ }^{41,42}$

Since the two transition temperatures are different, we must scale the respective temperatures in order to make a comparison. It has previously been shown that for relatively flexible chain polymers such as poly(propylene oxide), ${ }^{43}$ poly(dimethylsiloxane), ${ }^{44}$ and poly(methylphenysiloxane) ${ }^{14}$ the fragility is invariant to chain length. On the other hand, for more rigid chain

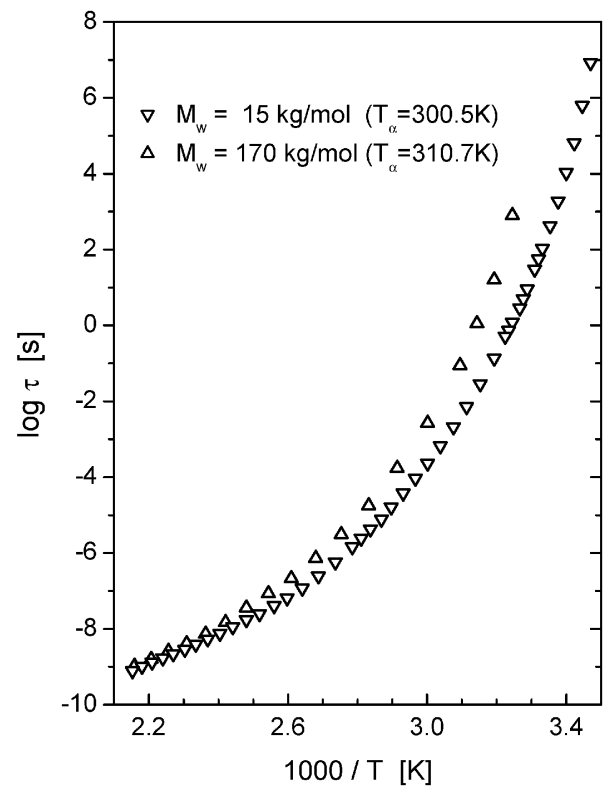

Figure 1. Dielectric relaxation times at atmospheric pressure for PVAc having a molecular weight equal to $15000 \mathrm{~g} / \mathrm{mol}$ $(\Delta$, from refs 37 and 38 ) and $170000 \mathrm{~g} / \mathrm{mol}(\nabla$, from ref 39 ). The 10 deg difference in the transition temperatures gives rise to as much as a 60-fold difference in $\tau$ at lower temperature.

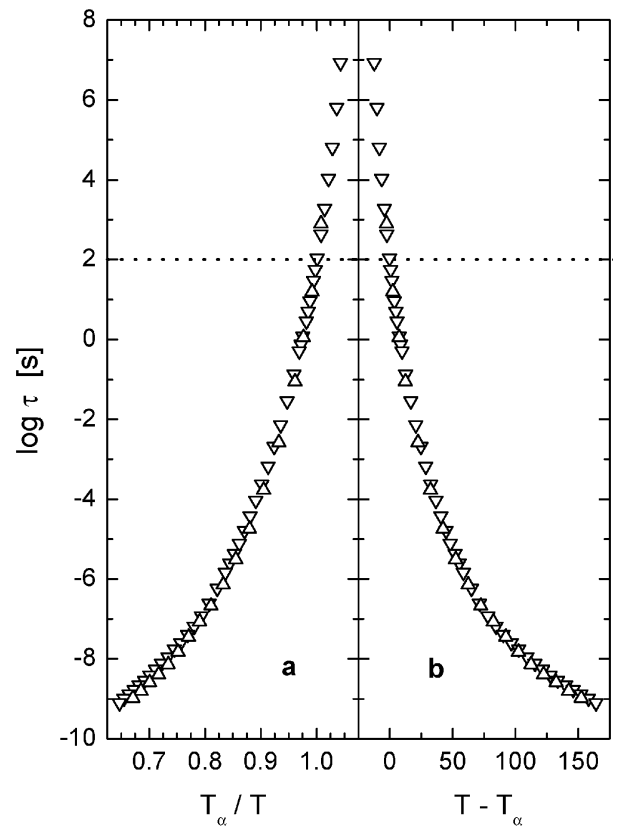

Figure 2. Dielectric relaxation times at atmospheric pressure for PVAc having respective molecular weights equal to 15000 $(\triangle)$ and $170000 \mathrm{~g} / \mathrm{mol}(\nabla)$ plotted (a) as a function of inverse temperature normalized by the temperature at which $\tau=100$ $s$ and (b) as a function of the difference between the measurement temperature and this characteristic temperature.

structures, such as polystyrene, ${ }^{45} \mathrm{~m}$ increases with molecular weight, due to the effect of chain ends. ${ }^{46}$ In Figure $2 \mathrm{a}$, the relaxation times of the two PVAc are plotted vs the reciprocal of temperature normalized by $\mathrm{T}_{\alpha}$. As can be seen, this superimposes the two sets of data, which means that the fragility is invariant to mol ecular weight. From the slope of the data in Figure $2 a$, we obtain $m=88 \pm 3$. This is close to previously reported values for this polymer. 40,47

An al ternative method of scaling the relaxation times is shown in Figure 2b, where $\tau$ is plotted as a function of the $T-T_{\alpha}$. Note that if the data fall on a single curve 


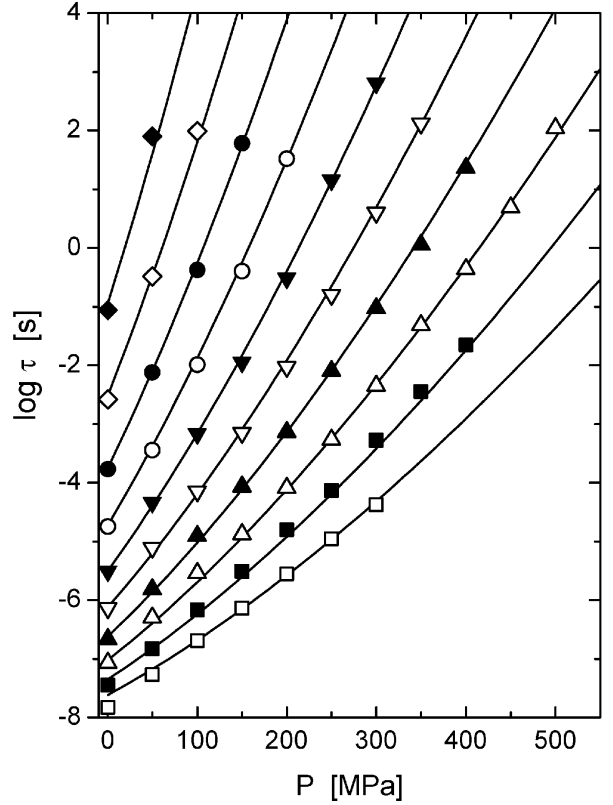

Figure 3. Dielectric relaxation times of PVAc as a function of pressure as reported by Heinrich and Stoll. ${ }^{39}$ The isotherms correspond to $\mathrm{T}=323.2(\diamond), 333.2(\diamond), 343.2(\bullet), 353.2(0)$ $363.2(\boldsymbol{\nabla}), 373.2(\nabla), 383.2(\mathbf{\Delta}), 393.2(\Delta), 403.2(\boldsymbol{\nabla})$, and 413.2 $K(\square)$. The solid curves through the data are the fits of eq 1.

vs $T_{\alpha} / T$, in principle they cannot al so have the same ( $T$ $-\mathrm{T}_{\alpha}$ ) dependence. Nevertheless, within the scatter in the data, the curves in Figure 2 superpose equally well. At least for these measurements, no distinction can be made between the two scalings, derived respectively from configurational entropy and free volume arguments.

Pressure Dependence of $\boldsymbol{\tau}$. In Figure 3 we show the data of Heinrich and Stoll 39 for PVAc at pressures up to $500 \mathrm{MPa}$ and temperatures up to $413 \mathrm{~K}$. At higher temperatures, their results at high pressure exhibit a curvature which we believe reflects systematic error; thus, these data are not analyzed herein. Previous workers have measured $\tau(\mathrm{P})$ for PVAc over a more limited frequency range at pressures below $300 \mathrm{MPa} .3,48,49$ Over this smaller range, the relaxation times can be taken to be proportional to exp $\mathrm{P}$, yielding an activation volume defined as $\Delta \mathrm{V}=2.303 \mathrm{RT}\left(\partial \log \tau_{\alpha} / \partial \mathrm{P}\right)_{\mathrm{T}}$. O'Reilly ${ }^{3}$ reported activation volumes ranging from 130 to 200 $\mathrm{mL} / \mathrm{mol}$ for temperatures from 390 to $340 \mathrm{~K}$. These values are ca. $5 \%$ lower than deduced from the data in Figure 3. However, as the broader range of pressure makes evident, a description of the pressure dependence as a simple volume activated process is only an approximation.

Since the data in Figure 3 are not linear, we employ the Avramov equation ${ }^{24}$

$$
\tau=\tau_{0} \exp \left[30\left(\frac{\mathrm{T}_{\mathrm{R}}}{\mathrm{T}}\right)^{\alpha}\left(1+\frac{\mathrm{P}}{\mathrm{P}_{\mathrm{R}}}\right)^{\beta}\right]
$$

in which $\tau_{0}, \mathrm{~T}_{\mathrm{R}}, \mathrm{P}_{\mathrm{R}}, \alpha$, and $\beta$ are material constants. The factor of 30 arises from the derivation of eq 1 in terms of the viscosity, which assumes a value close to $\exp (30)$ poise near the glass temperature. It is retained herein for consistency with the literature. The Avramov model assumes activated transport over a random distribution of energy barriers as the mechanism for structural relaxation. Explicit consideration of volume effects is

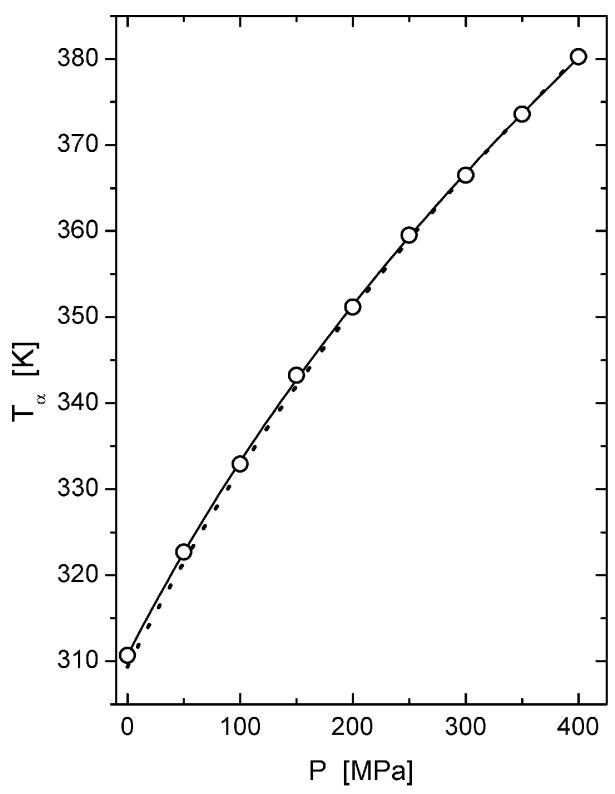

Figure 4. Temperature at which $\tau=100 \mathrm{~s}(0)$ along with the best-fit of eq 2 (solid line). The dashed line represents eq 2 using the parameters obtained from fitting the $\tau(\mathrm{P})$ data in Figure 3.

absent, although the energy barriers include intermolecular constraints, and thus volume contributions are not necessarily negated by the model.

Equation 1 has been shown to describe $\tau(P, T)$ for both molecular glass formers ${ }^{50,51}$ and polymers, ${ }^{14,52-54}$ provided there is no change of the temperature dependence with pressure $^{55}$ Although some materials exhibit a change in fragility with pressure, ${ }^{17,23,43,52,56} \mathrm{~m}(\mathrm{P})$ for PVAC is constant. ${ }^{56} \mathrm{U}$ sing the best-fit values of $\tau_{0}=(9.37$ $\pm 0.33) \times 10^{-10} \mathrm{~s}, \alpha=7.11 \pm 0.20, \beta=1.62 \pm 0.01, \mathrm{~T}_{\mathrm{R}}$ $=302.2 \pm 0.3 \mathrm{~K}$, and $\mathrm{P}_{\mathrm{R}}=271 \pm 4 \mathrm{MPa}$, we calculate the curves shown in Figure 3, which satisfactorily describe the experimental data over this range of pressure and temperature.

Rearrangement of eq 1 yields the pressure dependence of $\mathrm{T}_{\alpha}$ as

$$
\mathrm{T}_{\alpha}=\left(\frac{30 \log \mathrm{e}}{\log \left(\tau\left(\mathrm{T}_{\alpha}\right)\right)-\log \left(\tau_{0}\right)}\right)^{1 / \alpha}\left(1+\frac{\mathrm{P}}{\mathrm{P}_{\mathrm{R}}}\right)^{\beta / \alpha} \mathrm{T}_{\mathrm{R}}
$$

Note that this expression is identical to the empirical Andersson equation, 16 commonly used to describe the pressure dependences of the transition temperature. ${ }^{17,52,57,58}$ I Figure 4, the experimental $\mathrm{T}_{\alpha}(\equiv \mathrm{T}(\tau=$ 100 s)) are plotted vs pressure, along with eq 2 using the parameters deduced from fitting the data in Figure 3. The latter deviate somewhat from the curve obtained by fitting eq 2 directly to the $T_{\alpha}$ data. This reflects some deviation of the experimental $\tau(\mathrm{P})$ from the Avramov equation. In the limit of zero pressure, we obtain $\mathrm{dT}_{\alpha} / \mathrm{dP}=0.25 \mathrm{~K} / \mathrm{MPa}$. This falls within the range of literature values for the pressure coefficient of the transition temperature of PVAc, obtained by various methods. ${ }^{3,5,59}$

In terms of $\mathrm{T}_{\alpha}$ and the Avramov parameters, the fragility is given by $m=\alpha\left(\log \left(\tau\left(T_{\alpha}\right)-\log \tau_{0}\right)\right.$, yielding $m=78 \pm 4$ herein. This value, calculated from all 10 isotherms in Figure 3, is about $10 \%$ smaller than the fragility obtained by restricting the analysis to the atmospheric temperature data in Figure 1. 
Although the Avramov equation is useful for describing simultaneously the temperature and pressure dependences, the model has a theoretical basis, with the parameters in eq 1 related to measurable thermodynamic properties. To evaluate these, we make use of PVT measurements of McKinney and Simha, ${ }^{5}$ who expressed the specific volume of amorphous PVAc above $\mathrm{T}_{\mathrm{g}}$ in terms of the Tait equation 60

$$
\mathrm{V}(\mathrm{T}, \mathrm{P})=\mathrm{V}(\mathrm{T}, 0)[1-0.0894 \ln (1+\mathrm{P} / \mathrm{B}(\mathrm{T}))]
$$

whereby the volume (in $\mathrm{mL} / \mathrm{g}$ ) at zero pressure $(\approx$ atmospheric) is given by

$$
V(T)=0.82485+5.855 \times 10^{-4} \mathrm{~T}+2.82 \times 10^{-7} \mathrm{~T}^{2}
$$

and the pressure coefficient is

$$
B(T)=2.0354 \times 10^{3} \exp \left(-4.257 \times 10^{-3} T\right)
$$

Note that, by convention, $\mathrm{T}$ is in ${ }^{\circ} \mathrm{C}$ in these equations. The Avramov parameters are related according to 24

$$
\mathrm{P}_{\mathrm{R}}=\frac{\mathrm{C}_{\mathrm{p}}}{\kappa_{0} \mathrm{~V}_{\mathrm{m}}} \frac{\alpha}{\beta}
$$

where $\mathrm{R}$ is the gas constant, $\mathrm{C}_{\mathrm{p}}$ the heat capacity, $\kappa_{0}$ the volume expansion coefficient, and $V_{m}$ the molar volume (the last three quantities evaluated at zero pressure). At $\mathrm{T}=373 \mathrm{~K}$ (the midrange of the data in Figure 3) and ambient pressure, eq 4 yields $\kappa_{0}=7.24 \times$ $10^{-4} \mathrm{~K}^{-1}$ and $\mathrm{V}_{\mathrm{m}}=76.2 \mathrm{~mL} / \mathrm{mol}$. The heat capacity of PVAc at this temperature and atmospheric pressure is $163 \mathrm{~J} /(\mathrm{mol} \mathrm{K}){ }^{41}$ Inserting these in eq 6, along with the values obtained for $\alpha$ and $\beta$ from Figure 3 , yields $\mathrm{P}_{\mathrm{R}}=$ $74 \mathrm{MPa}$. This is more than a factor of 3 smaller than the $P_{R}$ determined from fitting the pressure dependence of the relaxation times.

The Avramov parameter $\mathrm{P}_{\mathrm{R}}$ can also be related to the pressure dependence of the thermal expansion coefficient ${ }^{24}$

$$
\kappa(\mathrm{P})=\frac{\kappa_{0} \mathrm{P}_{\mathrm{R}}}{\mathrm{P}_{\mathrm{R}}+\mathrm{P}}
$$

Using the value of $P_{R}$ obtained from fitting the relaxation times in Figure 3, we see in Figure 5 that eq 7 overpredicts the pressure dependence of $\kappa$; that is, the value of $\mathrm{P}_{\mathrm{R}}$ is too small to account for $\kappa(\mathrm{P})$, by almost $40 \%$. Thus, testing the Avramov model, beyond its ability to fit $\tau(T, P)$ data, reveals discrepancies. The bestfit value of $P_{R}$ is too large according to eq 6 and too small per eq 7. From these results, it is clear that a physical interpretation of the Avramov parameters in eq 1 is problematic, at least for PVAc.

Relative Contributions of Temperature and Volume to $\boldsymbol{\tau}$. Pressure alters the relaxation times of a supercooled liquid by changing the volume (density), whereas temperature alters $\tau$ by changing the thermal energy as well as the volume. This is shown in Figure 6 , wherein the relaxation times from Figure 3 are plotted as a function of volume (the latter from eqs 3-5). The relative contribution of temperature and volume can be quantified by comparison of the temperature dependence of $\tau$ at constant volume to that at constant pressure, expressed in terms of the respective activation energies for isochoric, $E_{V}=\left.R\left(\partial \ln \tau / \partial T^{-1}\right)\right|_{v}$, and isobaric,

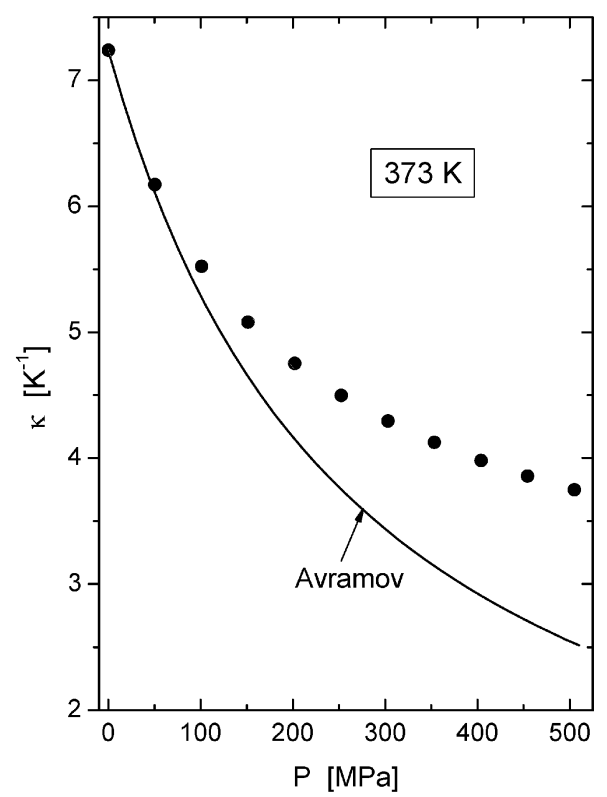

Figure 5. Thermal expansion coefficient of PVAc as a function of pressure (๑). The solid curve represents eq 7, using $P_{R}$ $(=275.3 \mathrm{MPa})$ determined from fitting the $\tau(\mathrm{P})$ in Figure 3.

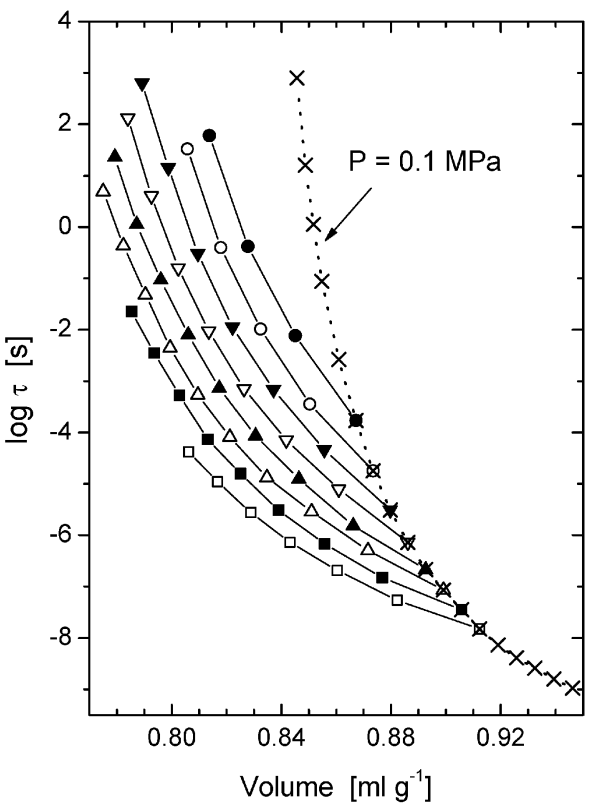

Figure 6. Volume dependence of the relaxation times as a function of temperature at constant atmospheric pressure $(x)$ and with varying pressure at constant temperature. The symbols for the latter are as defined in Figure 3.

$\mathrm{E}_{\mathrm{P}}=\left.\mathrm{R}\left(\partial \mathrm{In} \tau / \partial \mathrm{T}^{-1}\right)\right|_{\mathrm{P}}$ conditions. ${ }^{27,61}$ (These are apparent activation energies, since $\log \tau$ is never directly proportional to reci procal temperature.) A value of $E_{v} / E_{p}$ equal to unity would reflect thermally activated molecular motion, with a negl igible ratio indicating the dominance of volume.

From the data in Figure 6, we obtain the relaxation times for any arbitrary fixed volume, with results shown as a function of temperature for three volumes $(0.852$ $\geq \mathrm{V} \geq 0.847 \mathrm{~mL} / \mathrm{g}$ ) in Figure 7. Also included are the dielectric relaxation times at (constant) atmospheric pressure. The isochoric $\tau(T)$ curves intersect the isobaric data at values of the relaxation time equal to 1,10 , and $100 \mathrm{~s}$. F rom the slopes of the curves at these loci, which are in the vicinity of the glass transition, $\mathrm{E}_{\mathrm{V}} / \mathrm{E}_{\mathrm{P}} \approx 0.6$. This indicates that thermal energy and volume both 


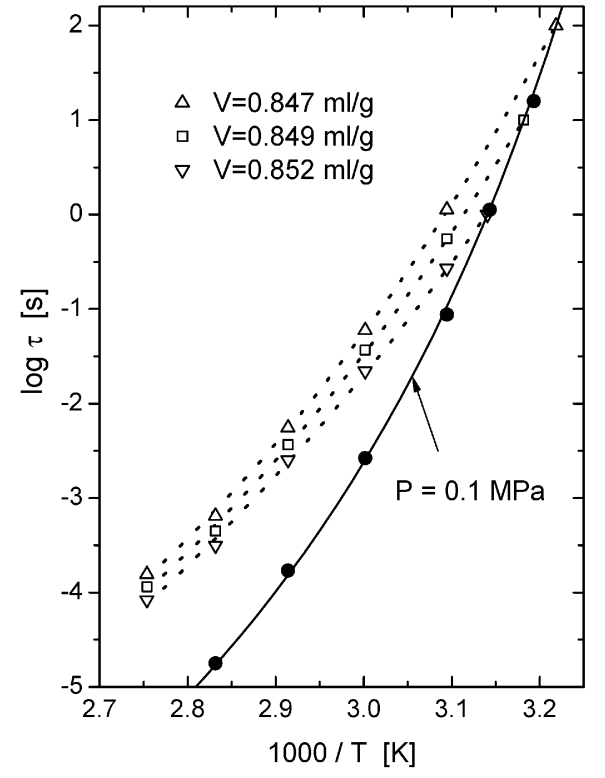

Figure 7. Temperature dependence of the dielectric relaxation times at atmospheric pressure $(\bullet)$ and at the indicated constant volumes, chosen to intersect the isobaric curve at $\tau$ $=1 \mathrm{~s}(\mathrm{~V}=0.852 \mathrm{~mL} / \mathrm{g}, \nabla), 10 \mathrm{~s}(\mathrm{~V}=0.849, \square)$, and $100 \mathrm{~s}(\mathrm{~V}=$ $0.847 \mathrm{~mL} / \mathrm{g}, \Delta)$. The slopes at the intersection of the isobaric and isochoric lines yield values for the respective apparent activation energies at constant pressure and constant volume: $\mathrm{E}_{\mathrm{a}}=437$ and $250 \mathrm{~kJ} / \mathrm{mol}(\tau=1 \mathrm{~s}), \mathrm{E}_{\mathrm{a}}=490$ and 293 $\mathrm{kJ} / \mathrm{mol}(\tau=10 \mathrm{~s})$, and $\mathrm{E}_{\mathrm{a}}=553$ and $330 \mathrm{~kJ} / \mathrm{mol}(\tau=100 \mathrm{~s})$.

exert a significant influence on the dynamics of supercooled PVAc.

The ratio of apparent activation energies can also be evaluated from 62

$$
E_{\mathrm{V}} / \mathrm{E}_{\mathrm{P}}=1-\gamma\left(\frac{\partial \mathrm{T}}{\partial \mathrm{P}}\right)_{\tau}
$$

where the thermal pressure coefficient, $\gamma=(\partial \mathrm{P} / \partial \mathrm{T}))_{V}$, is calculated from the PVT data (eq 3). For $\mathrm{V}=0.85 \mathrm{~mL} /$ $\mathrm{g}$, we obtain $\gamma=1.31 \mathrm{MPa} / \mathrm{K}$. The quantity $(\partial \mathrm{T} / \partial \mathrm{P})_{\tau}$ evaluated at $\tau=100 \mathrm{~s}$ is just the pressure dependence of the glass transition, $\mathrm{dT} / \mathrm{dP}=0.25 \mathrm{~K} / \mathrm{MPa}$. Thus, from eq 8 we calculate that $E_{v} / E_{p}=0.67$, which is comparable to the value of this ratio deduced from Figure 7. This equivalence is a tautology, since the two calculations derive from the same experimental data.

An alternative means of gauging the relative influence of temperature and pressure, suggested by Ferrer et $\mathrm{al}_{.,}{ }^{28}$ is from the ratio of the thermal expansion coefficient at constant pressure, $\alpha_{P}=-\rho^{-1}(\partial \rho / \partial T)_{P}$, to the coefficient of thermal expansivity at constant value of the relaxation time, $\alpha_{\tau}=-\rho^{-1}(\partial \rho / \partial \mathrm{T})_{\tau}$. A value of $\left|\alpha_{\tau}\right| /$ $\alpha_{p} \gg 1$ implies thermally activated dynamics, whereas this ratio is cl oser to unity if volume plays an important role.

The densities at atmospheric pressure, calculated from the PVT data (eq 4), are shown in Figure 8. Also displayed are the densities, calculated using eqs 1 and 3 , for pressures at which $\tau=100 \mathrm{~s}$. From the slopes of the two lines, we obtain $\left|\alpha_{\tau}\right| / \alpha_{p}=1.8$. This ratio is unchanged if the calculation is done at a higher temperature, for example corresponding to $\tau=1 \mathrm{~s}$ (Figure 8). Consistent with the analysis of the apparent activation energies, the small magnitude of $\left|\alpha_{\tau}\right| / \alpha_{p}$ indicates that both temperature and volume significantly influence the dynamics of PVAc near its glass transition.

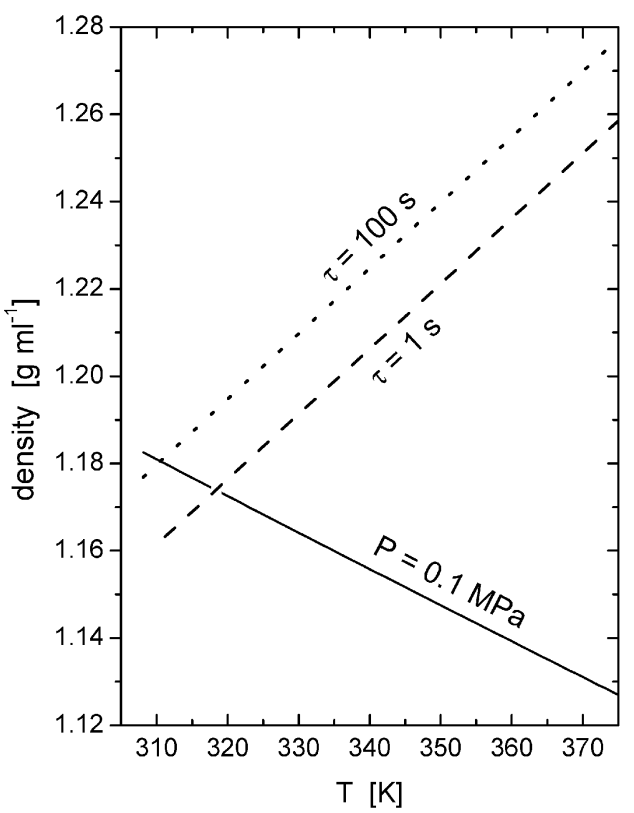

Figure 8. Mass density of PVAc as a function of temperature for atmospheric pressure (solid line), yielding the isobaric thermal expansion coefficient, $\alpha_{p}=7.033 \times 10^{-4} \mathrm{~K}^{-1}$, and calculated for the pressures at which the relaxation time equals $1 \mathrm{~s}$ (dashed line) and $100 \mathrm{~s}$ (dotted line). The isochronic lines are parallel, yielding $\alpha_{\tau}=-1.271 \times 10^{-3} \mathrm{~K}^{-1}$.

\section{Summary and Concluding Remarks}

Comparing dielectric relaxation times for two PVAc, we find that fragility is independent of molecular weight, for $\mathrm{M}_{\mathrm{w}}$ low enough to depress $\mathrm{T}_{\mathrm{g}}$ by $10 \mathrm{deg}$. The fragility is also invariant to pressure; thus, we can employ the Avramov model to analyze the combined $T$ and $\mathrm{P}$ dependences. Although the model accurately describes the experimental isotherms for $\mathrm{P} \leq 500 \mathrm{MPa}$, the parameters obtained by fitting the $\tau(\mathrm{T}, \mathrm{P})$ data are not consistent with the values independently calculated from thermodynamic quantities. Thus, the Avramov model's utility herein is limited to parametrization of the experimental results.

Making use of published PVT measurements for PVAc, we express the dielectric relaxation times as a function of volume. From these data, the isochoric and isobaric (apparent) activation energies are calculated. The ratio of the two activation energies is $\sim 0.6$, which indicates that both temperature and volume influence the magnitude of the relaxation times. This conclusion is supported by the ratio of the thermal expansion coefficients, $\left|\alpha_{\tau}\right| / \alpha_{p}=1.8$.

In Table 1 we compare our findings for PVAc to results for other glass-formers, both molecular6,28,32-34,51,62 and polymeric. ${ }^{33,54,63,64}$ The general observation is that for van der Waals liquids neither temperature nor pressure is the dominant variable controlling $\tau$. For polymers such as PVAc and poly(methyl acrylate), polar interactions may increase somewhat the role of temperature; however, it is only for the polyal cohols in Table 1 , associated with extensive hydrogen bonding, that the effect of volume can be neglected. ${ }^{33}$

The reduced efficacy of volume in $\mathrm{H}$-bonded liquids reflects two competing effects: ${ }^{33,66} \mathrm{As}$ pressure increases, molecular crowding, and consequently stronger intermolecular cooperativity and more heterogeneous dynamics, retard structural relaxation. However, orientation-specific interactions, such as $\mathrm{H}$-bonding, are suppressed by pressure, ${ }^{67-69}$ which has the effect of 
Table 1. Relative Contribution of Density and Temperature to the Relaxation Times near $\mathbf{T}_{g}$ for Various Glass-Forming Liquids and Polymers ${ }^{a}$

\begin{tabular}{lccccl}
\hline & $\mathrm{T}_{\mathrm{g}}[\mathrm{K}]$ & $\left|\alpha_{\tau}\right| / \alpha_{\mathrm{p}}$ & $\mathrm{E}_{\mathrm{V}} / \mathrm{E}_{\mathrm{P}}$ & $\mathrm{m}$ & ref \\
\hline BMPC & 243 & 0.58 & 0.39 & 72 & 34 \\
BMMPC & 263 & 0.72 & 0.41 & 66 & 34 \\
salol & 220 & & 0.43 & 73 & 51 \\
KDE & 313 & 0.98 & & 73 & 6 \\
PDE & 249 & 1.25 & 0.53 & 96 & 33 \\
o-terphenyl & 244 & & 0.55 & 81 & 2,62 \\
poly(propylene oxide) & 198 & & $(0.55)^{\mathrm{b}}$ & 74 & 47,63 \\
diglycidyl ether of & 335 & 1.8 & 0.6 & 95 & 54 \\
$\quad$ bisphenol A & & & & & \\
PVAc & 311 & 1.8 & 0.6 & 78 & herein \\
PPGE & 258 & 1.7 & 0.63 & 95 & 33,65 \\
poly(methyl acrylate) & 276 & & $(0.78)^{\mathrm{c}}$ & 102 & 2,64 \\
sorbitol & 273 & 6 & 0.87 & 128 & 32 \\
glycerol & 189 & 17 & & 54 & 17,28
\end{tabular}

a BMPC $\equiv 1,1^{\prime}$-bis(p-methoxyphenyl)cyclohexane, BMMPC $\equiv$ 1,1'-di(4-methoxy-5-methylphenyl)cyclohexane, KDE $\equiv$ cresolphthalein dimethyl ether, PDE $\equiv$ phenylphthalein dimethyl ether, and PPGE $\equiv$ for poly(phenyl glycidyl ether)-co-formaldehyde. ${ }^{b} \mathrm{At}$ $\mathrm{T}=\mathrm{T}_{\mathrm{g}}+35 .{ }^{\mathrm{c}} \mathrm{At} \mathrm{T}=\mathrm{T}_{\mathrm{g}}+31$.

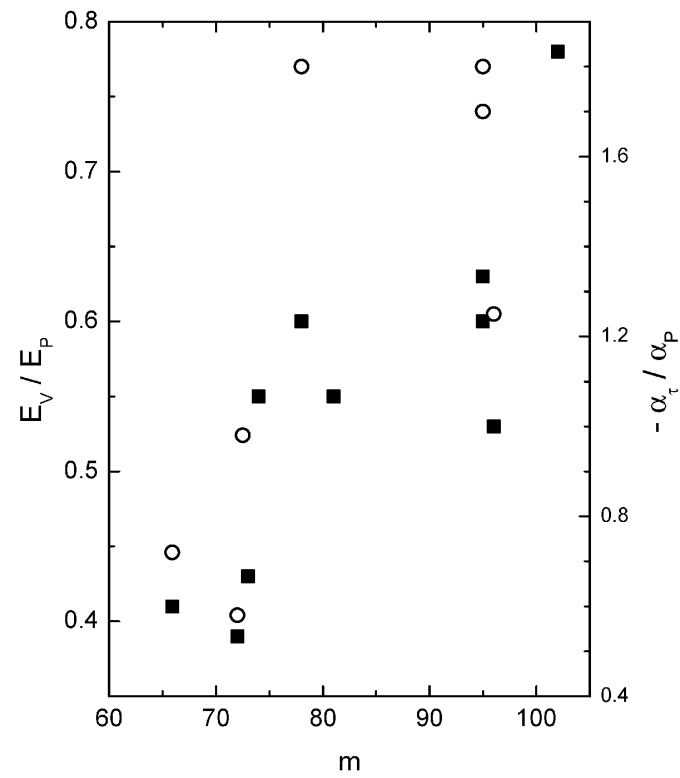

Figure 9. Relationship between fragility and the two parameters characterizing the relative contribution of temperature and density to the relaxation times for the nonassociated glassformers in Table $1\left(E_{v} / E_{P}, \mathbf{\square} ;\left|\alpha_{\tau}\right| / \alpha_{P}, O\right)$.

enhancing molecular mobility. Temperature effects, on the other hand, reinforce one another; reduced thermal energy slows molecular mobility both directly and by promoting specific interactions. The consequence is that for associated liquids in the supercooled regime temperature dominates the dynamics.

Whereas the magnitude of $E_{\vee}$ obviously is determined by the contribution of thermal energy to the relaxation times, it has been suggested that nonlinearity in a plot of $\log \tau$ vs $1 / \mathrm{T}$ at constant density also reflects solely the effect of temperature. 28,70 This idea is not borne out the results in Figure 6 , in which significant curvature is observed in the constant density curves. For the materials in Table 1, we can compare the fragility, which measures the activation energy at constant pressure, to the magnitude of the volume contribution to $\tau$. Values of $m$ collected from the literature are listed in Table 1 and plotted vs $E_{V} / E_{P}$ and $\left|\alpha_{\tau}\right| / \alpha_{P}$ in Figure 9. We exclude the hydrogen-bonded polyalcohols. From these results, it is tempting to suggest that for van der
Waals glass-formers a weak general trend may exist between more thermally activated dynamics and greater deviation from non-Arrhenius behavior.

Acknowledgment. This work was supported by the Office of Naval Research.

\section{References and Notes}

(1) Plazek, D. J .; Ngai, K. L Macromolecules 1991, 24, 1222.

(2) Bohmer, R.; Ngai, K. L.; Angell, C. A.; Plazek, D. J . J . Chem Phys. 1993, 99, 4201.

(3) O'Reilly, J. M. J . Polym. Sci. 1970, 57, 429.

(4) Questad, D. L.; Pae, K. D.; N ewman, B. A.; Scheinbeim, J . I. J. Appl. Phys. 1980, 51, 5100.

(5) McKinney, J. K.; Simha, R. Macromolecules 1974, 7, 894

(6) Paluch, M.; Roland, C. M.; Best, A. J . Chem. Phys. 2002, 117, 1188.

(7) Angell, C. A. J . Non-Cryst. Solids 1991, 131-133, 13.

(8) Ferry, J . D. Viscoelastic Properties of Polymers, 3rd ed.; Wiley: New York, 1980

(9) Floudas, G.; Gravalides, C.; Reisinger, T.; Wegner, G. J Chem. Phys. 1999, 111, 9847.

(10) Roland, C. M.; Ngai, K. L. Macromolecules 1991, 24, 5315; 1992, 25, 1844

(11) Ngai, K. L.; Roland, C. M. Macromolecules 1993, 26, 6824

(12) Roland, C. M. Macromolecules 1994, 27, 4242.

(13) Fytas, G.; Dorfmuller, Th.; Wang, C. H.J . Phys. Chem. 1983 $87,50411$.

(14) Paluch, M.; Roland, C. M.; Pawlus, S. J . Chem. Phys. 2002 116, 10932.

(15) Paluch, M.; Hensel-Bielowka, S.; Psurek, T. J . Chem. Phys. 2000, 113, 4374.

(16) Andersson, S. P.; Andersson, O. Macromolecules 1998, 31 2999.

(17) Paluch, M.; Casalini, R.; Hensel-Bielowka, S.; Roland, C. M. J. Chem. Phys. 2002, 116, 9839.

(18) Cohen, M. H.; Grest, G. S. Phys. Rev. B 1979, 20, 1077. Grest, G. S.; Cohen, M. H. Adv. Chem. Phys. 1981, 48, 455.

(19) Corezzi, S.; Capaccioli, S.; Casalini, R.; Fioretto, D.; Paluch, M.; Rolla, P. A. Chem. Phys. Lett. 2000, 320, 113.

(20) Paluch, M.; Casalini, R.; Roland, C. M. Phys. Rev. E, in press

(21) Bendler, J. T.; Fontanella, J .; Schlesinger, M. Phys. Rev. Lett. 2001, 87, 195503.

(22) Casalini, R.; Capaccioli, S.; Lucchesi, M.; Rolla, P. A.; Corezzi S. Phys. Rev. E 2001, 63, 31207. Casalini, R.; Capaccioli, S.; Lucchesi, M.; Rolla, P. A.; Paluch, M; Corezzi, S.; Fioretto, D. Phys. Rev. E 2001, 64, 041504. Capaccioli, S.; Lucchesi, M.; Casalini, R.; Presto, S.; Rolla, P. A.; Viciosa, M. T.; Corezzi, S.; Fioretto, D. Philos. Mag. B 2002, 82, 651.

(23) Casalini, R.; Paluch, M.; Roland, C. M. Phys. Rev. E, submitted.

(24) Avramov, I.; Milchev, A.J . Non-Cryst. Solids 1988, 104, 253. Avramov, I. J . Non-Cryst. Solids 2000, 262, 258.

(25) Williams, M. L.; Landel, R. F.; Ferry, J . D. J . Am. Chem. Soc. 1955, 77, 3701

(26) Turnbull, D.; Cohen, M. J . Chem. Phys. 1970, 52, 3038.

(27) Williams, G. In Dielectric Spectroscopy of Polymeric Materials; Runt, J. P., Fitzgerald, J. J., Eds.; American Chemical Society: Washington, DC, 1997.

(28) Ferrer, M. L.; Lavrence, Ch.; Demirjian, B. G.; Kivelson, D.; Alba-Simonesco, Ch.; Tarjus, G. J . Chem. Phys. 1998, 109 8010.

(29) Alba-Siminoesco, C.; Kivelson, D.; Tarjus, G. J . Chem. Phys. 2002, 116, 5033

(30) Pakula, T.J . Mol. Liq. 2000, 86, 109.

(31) Macedo, P. B.; Litovitz, T. A. J . Chem. Phys. 1965, 42, 245

(32) Hensel-Bielowka, S.; Paluch, M.; Roland, C. M. J . Phys. Chem. B 2002, 106, 12459

(33) Paluch, M.; Casalini, R.; Roland, C. M. Phys. Rev. B 2002 66, 092202.

(34) Paluch, M.; Casalini, R.; Roland, C. M.; Meier, G.; Patkowski, A. J . Chem. Phys., in press.

(35) MacKenzie, J . D. J . Chem. Phys. 1958, 28, 1037.

(36) McCrum, N. G.; Read, B. E.; Williams, G. Anelastic and Dielectric Effects in Polymeric Solids; Wiley: London, 1967; Dover: New York, 1991.

(37) Stickel, F. Doctoral Thesis, 1995, J ohannes GutenbergUniverstat Mainz, Germany (Shaker, Achen, 1995).

(38) Richert, R. Physica A 2000, 287, 26.

(39) Heinrich, W.; Stoll, B.Colloid Polym. Sci. 1985, 263, 873.

(40) Ngai, K. L.; Roland, C. M. Polymer 2002, 43, 567. 
(41) Gaur, U.; Wunderlich, B. B.; Wunderlich, B. J . Phys. Chem. Ref. Data 1983, 12, 29.

(42) McKinney, J. E.; Simha, R. Macromolecules 1976, 9, 430.

(43) Roland, C. M.; N gai, K. L. Macromolecules 1992, 25, 5765

(44) Roland, C. M.; N gai, K. L. Macromolecules 1996, 29, 5747.

(45) Santangel o; P. G.; Roland, C. M. Macromolecules 1998, 31, 4581.

(46) Santangelo, P. G.; Roland, C. M.; Chang, T.; Roovers, J. Macromol ecules 2001, 34, 9002.

(47) Roland, C. M.; Santangelo, P. G.; Ngai, K. L. J . Chem. Phys. 1999, 111, 5593.

(48) Saito, S.; Sasabe, H.; Nakajima, T.; Yada, K. J . Polym. Sci., Part A2 1968, 6, 1297.

(49) Questad, D. L.; Oskooie-Tabrizi, M. J . Appl. Phys. 1982, 53, 6574.

(50) Paluch, M.; Ngai, K.; Hensel-Bielowka, S. J . Chem. Phys. 2001, 114, 10827.

(51) Casalini, R.; Roland, C. M.; Paluch, M. J . Phys. Chem. B, submitted.

(52) Paluch, M.; Pawlus, S.; Roland, C. M. Macromolecules 2002, 35, 7338.

(53) Paluch, M.; Psurek, T.; Roland, C. M. J . Phys.: Condens. Matter 2002, 14, 9489.

(54) Paluch, M.; Roland, C. M.; Gapinski, J .; Patkowski, A. J . Chem. Phys., in press.

(55) Paluch, M.; Roland, C. M. J . Non-Cryst. Solids 2003, 316, 413.
(56) Huang, D.; Colucci, D. M.; McKenna, G. B. J . Chem. Phys. 2002, 116, 3925; 2002, 117, 7390.

(57) Bendler, J . T.; Fontanella, J . J .; Scheslinger, M. F.; Wintersgill, M. C. Electrochim. Acta, submitted.

(58) Ziolo, J .; Paluch, M.; Roland, C. M. J . Chem. Phys. 2002, 117, 2317.

(59) Atake, T.; Angell, C. A. J . Phys. Chem. 1979, 83, 3218.

(60) Tait, P. G. Physics and Chemistry of the Voyage of H. M. S Challenger; HMSO: London, 1888; Vol. 2, Part 4.

(61) Williams, G. Trans. Faraday Soc. 1964, 60, 1556.

(62) Naoki, M.; Endou, H.; Matsumoto, K. J . Phys. Chem. 1987, 91, 4169.

(63) Williams, G. Trans. Faraday Soc. 1965, 61, 1564.

(64) Williams, G. Trans. Faraday Soc. 1964, 60, 1548.

(65) Paluch, M.; Hensel-Bielowka, S.; Ziolo, J. Phys. Rev. E 2000, 61, 526.

(66) Roland, C. M.; Casalini, R.; Paluch, M. Chem. Phys. Lett. 2003, 367, 259

(67) Poole, P. H.; Sciortino, F.; Grande, T.; Stanley, H. E.; Angell, C. A. Phys. Rev. Lett. 1994, 73, 1632.

(68) Naoki, M.; Katahira, S. J . Phys. Chem. 1991, 95, 431.

(69) Cook, R. E.; King, H. E.; Peiffer, D. G. Phys. Rev. Lett. 1992, 69, 30732.

(70) Ferrer, M. L.; Kivelson, D. J . Chem. Phys. 1999, 110, 10963.

MA025791Z 